\title{
Intranasal immunization with coxsackievirus A16 virus-like particles confers protection against lethal infection in neonatal mice
}

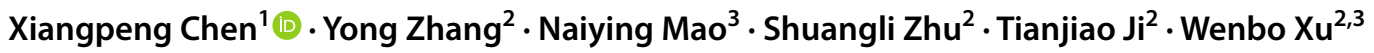

Received: 13 March 2019 / Accepted: 26 August 2019 / Published online: 30 September 2019

(c) Springer-Verlag GmbH Austria, part of Springer Nature 2019

\begin{abstract}
Coxsackievirus A16 (CV-A16) is one of the main causative agents of hand, foot and mouth disease (HFMD) in young children and has become prevalent in the Asia-Pacific region in recent years. However, no approved vaccines or drugs are available for CV-A16 infection. CV-A16 virus-like particles (VLPs) are a potential vaccine candidate; however, whether the intranasal route of immunization is suitable for inducing immune responses against CV-A16 infection has not been clarified. In this study, the comprehensive immunogenicity and protective efficacy of the CV-A16 VLP vaccine were evaluated by multiple methods in a mouse model. In mice, a high neutralizing antibody (NTAb) titre could be elicited by intranasal immunization with CV-A16 VLPs, which produced NTAb levels similar to those induced by intranasal immunization with inactivated CV-A16. Passive immunity with NTAbs provided very good protection, as the survival rate of the immunized neonatal mice was $100 \%$ after challenges with CV-A16 at a dose of $1000 \mathrm{LD}_{50}$. Passive protective effects were transferred to the neonates via the mother, thus protecting all the pups against challenges with the homologous or heterologous strains of CV-A16 at a dose of $1000 \mathrm{LD}_{50}$. In addition, intranasal immunization with CV-A16 VLPs also induced the production of mucosal secretory IgA (s-IgA) antibodies, which may inhibit CV-A16 virus invasion. This study provides valuable supplemental information to facilitate our understanding of the specific protective efficacy of CV-A16 VLPs and has significance for development of the candidate vaccine into a safe and effective vaccine.
\end{abstract}

Handling Editor: Tim Skern.

Wenbo Xu

wenbo_xu1@aliyun.com

1 Key Laboratory of Major Diseases in Children, Ministry of Education, National Clinical Research Center for Respiratory Diseases, Beijing Key Laboratory of Pediatric Respiratory Infection Diseases, Laboratory of Infection and Virology, Beijing Pediatric Research Institute, Beijing Children's Hospital, Capital Medical University, National Center for Children's Health, Beijing 100045, China

2 WHO WPRO Regional Polio Reference Laboratory and NHC Key Laboratory of Medical Virology and Viral Diseases, National Institute for Viral Disease Control and Prevention, Chinese Center for Disease Control and Prevention, No. 155 Chang-bai Road, Beijing 102206, China

3 WHO WPRO Regional Reference Measles/Rubella Laboratory and NHC Key Laboratory of Medical Virology and Viral Diseases, National Institute for Viral Disease Control and Prevention, Chinese Center for Disease Control and Prevention, Beijing 102206, China

\section{Introduction}

Hand, foot and mouth disease (HFMD) has recently become a common infectious disease in children in China. Enterovirus A71 (EV-A71) and coxsackievirus A16 (CV-A16) are still the most predominant pathogens in newly occurring HFMD cases [1, 2]. However, according to previous studies, the predominant pathogen of HFMD constantly changes, and alternation among the enteroviruses (EVs) always occurs [3-5]. CV-A16, CV-A6, CV-A10, and other EVs (non-EVA71) play a major role in HFMD, while a public immunity barrier against EV-A71 is established via natural infection or passive immunization [6-8]. In addition, neutralizing antibodies induced by EVs provide protective immunity for only their respective serotypes and are not expected to elicit heterotypic immunity against other serotypes; thus, the development of multivalent $\mathrm{EV}$ vaccines is necessary to prevent and control the spread of HFMD.

In addition to causing mild symptoms, CV-A16 infection can also result in myocarditis and pericarditis, acute heart failure, and even death $[9,10]$. Coinfection with CV-A16 and EV-A71 also increases the complexity of the disease. 
The HFMD epidemic could not be controlled solely by the EV-A71 vaccine. Therefore, the development of a CV-A16 vaccine is urgently required to ensure the effective prevention of HFMD. An inactivated CV-A16 vaccine is difficult to develop due to the poor yield of the virus in cell culture.

Virus-like particles (VLPs), consisting of recombinant structural proteins that mimic the structure and antigenicity of the parental infectious virus, have great potential as vaccine candidates. However, VLPs lack viral nucleic acids and are completely noninfectious [11]. As a safe and effective strategy for vaccine development, VLPs have been successfully used to vaccinate against human papilloma virus and hepatitis B virus for many years [12-15]. Recombinant EV-A71 VLPs have been shown to induce neutralizing antibodies and protect neonatal mice against a lethal challenge with EV-A71 [16, 17]. Recently, the CV-A16 VLP vaccine candidate was shown to induce the production of high neutralizing antibody titres after intraperitoneal (i.p.) administration and to provide effective protection against CV-A16 infection [18-20].

In this study, intranasal immunization was used to evaluate the immunogenicity of the CV-A16 VLP vaccine candidate in comparison with that of the inactivated whole virus. Furthermore, we assessed the immunoprotective effect of the neutralizing antibodies in a neonatal Institute of Cancer Research (ICR) mouse model by analysing viral load variation, lethal challenge, etc. This study serves as a solid foundation for research on and development of CV-A16 VLP vaccine candidates against CV-A16 infection in humans.

\section{Materials and methods}

\section{Preparation of CV-A16 VLPs}

CV-A16 VLPs were produced using a baculovirus expression system as described previously [20]. Briefly, Sf9 insect cells (Invitrogen Life Technologies) were infected with a recombinant baculovirus (AcMNPV-P1-3CD) coexpressing codon-optimized P1 (under polyhedrin promoter control) and $3 \mathrm{CD}$ (under CMV promoter control) of the CV-A16 521-01T strain. The VLPs were purified by continuous caesium chloride $(\mathrm{CsCl}$, Sinopharm Chemical Reagent Co., Ltd.) gradient ultracentrifugation for final purification [21].

The CV-A16 strains 521-01T (B1b genotype, isolated from throat swab specimens collected from an inpatient with HFMD, Shandong Province, China, 2007) and NJ1031 (B 1a genotype, isolated from throat swab specimens collected from an inpatient with HFMD, Jiangsu Province, China, 2010) were used for the challenge experiments. All viral titres were determined by the microtitration method and expressed in units of 50\% tissue culture infectious dose $\left(\mathrm{TCID}_{50}\right)$. The inactivated CV-A16 virus (521-01T strain) was produced as described previously [21].

The purified VLPs and inactivated viruses were examined by western blot and transmission electron microscopy. Protein concentrations were measured using a BCA protein assay kit (Thermo Fisher Scientific, USA) according to the manufacturer's instructions.

\section{Immunization of ICR mice}

Animal treatment and care were provided according to the guidelines of the Chinese Center for Disease Control and Prevention (Beijing, China). All animal studies were approved by the Animal Care and Welfare Committee of the National Institute for Viral Disease Control and Prevention, Chinese Center for Disease Control and Prevention.

VLPs, inactivated whole viruses and negative control were mixed with the adjuvant CpG-oligonucleotide (ODN) (TaKaRa Biotech Co., Ltd.) according to the manufacturer's instructions. Four groups (14 mice per group) of 6-week-old female ICR mice (Vital River Lab Animal Technology Co., Ltd, Beijing, China) were immunized intranasally with $5 \mu \mathrm{g}(50 \mu \mathrm{L}$ per mouse) of antigens, adjuvant or PBS at weeks 0,2 and 4. Mice in each group were divided into three cages containing six, five, and three mice, respectively. Blood samples were collected from the tails of three mice in each group at weeks 0,2 , $4,6,8,10,12$ and 14 for serum antibody measurement. Five mice from each group were sacrificed at week 6 for the detection of cellular immune responses and determination of serum neutralizing antibody titres; respiratory tract washes, vaginal washes and small-intestine samples were also collected for the assessment of s-IgA levels. The other six mice in each group were allowed to mate after the second immunization.

\section{CV-A16-specific antibody measurements}

CV-A16-specific serum IgG and s-IgA antibodies were measured by ELISA. In brief, ELISA plates were coated with inactivated CV-A16 (20 ng/well). Subsequently, the plates were incubated with serially diluted serum or mucosal secretion samples. A horseradish peroxidase (HRP)-conjugated goat anti-mouse IgG or IgA antibody (Abcam, Cambridge, MA, USA) was added as the secondary antibody. Tetramethylbenzidine substrate (100 $\mu \mathrm{L} /$ well, Sigma-Aldrich) was added for colour development. The absorbance was measured at $450 \mathrm{~nm}$ using an ELISA plate reader (Thermo Scientific Multiskan ${ }^{\mathrm{TM}}$ GO; Thermo Fisher Scientific Oy, Vantaa, Finland). 


\section{Neutralization assay}

Serum neutralization titres were determined based on a $\mathrm{TCID}_{50}$ reduction assay in rhabdomyosarcoma (RD) cells [22]. Briefly, $50 \mu \mathrm{L}$ of serially twofold diluted serum samples was mixed with $50 \mu \mathrm{L}\left(100 \mathrm{TCID}_{50}\right)$ of CV-A16 521$01 \mathrm{~T}$ and incubated at $37{ }^{\circ} \mathrm{C}$ for $2 \mathrm{~h}$. Subsequently, RD cells were added to the wells and incubated. Three days after infection, the cytopathic effect (CPE) was observed. The neutralization titre was determined as the highest serum dilution that prevented CPE in $>50 \%$ of the wells.

\section{ELISPOT assay}

Interferon (IFN)- $\gamma$ secretion by splenocytes was measured using an ELISPOT kit (Dakewe, Shenzhen, China). In brief, spleens were collected from three immunized mice from each group at two weeks after the last immunization. The splenocytes were isolated and counted. Precoated plates were blocked with complete RPMI 1640 medium for $10 \mathrm{~min}$. Fresh splenic cells $\left(2 \times 10^{5} /\right.$ well $)$ were added to the plates. Subsequently, CV-A16 VLPs or inactivated virus at a concentration of $15 \mu \mathrm{g} / \mathrm{mL}$ was added to stimulate the cells at $37^{\circ} \mathrm{C}$ for $36 \mathrm{~h}$, whereas phorbol myristoyl acetate (PMA) was added as a positive control. Subsequently, plates were processed in accordance with the manufacturer's instructions after removal of cells. Spot-forming cells (SFCs) were imaged and counted using a CTL ImmunoSpot reader (Cellular Technology Ltd, Shaker Heights, OH, USA).

\section{Mouse infection experiments}

To determine the $50 \%$ lethal dose $\left(\mathrm{LD}_{50}\right)$ and to investigate dose dependence, neonatal ICR mice less than $48 \mathrm{~h}$ of age (8-12 per group) were inoculated intracerebrally (i.c.) with serial tenfold dilutions of 521-01T or NJ10-31 $(1,1 \times 10$, $1 \times 10^{2}, 1 \times 10^{3}$ and $1 \times 10^{4} \mathrm{TCID}_{50} /$ mouse $)$. The mice in the control group were inoculated with cell culture maintenance medium. Mice were observed daily for clinical symptoms or death until 15 days post-inoculation (dpi). The control group remained healthy throughout the experiments. The $\mathrm{LD}_{50}$ was calculated by the Reed and Muench method [23].

\section{Viral loads in tissues of infant mice post-challenge}

The viral load in three control mice and 30 experimental mice after i.c. inoculation with culture maintenance medium or the $521-01 \mathrm{~T}$ virus (1000 $\mathrm{LD}_{50} /$ mouse) was determined as described by Mao et al. [24]. Tissues and blood samples from experimental mice $(n=3$, per time point) were collected aseptically and weighed at 1, 7, 24, $48,72,96$, and $120 \mathrm{~h}$ postinfection. Samples from three control mice were collected at $1 \mathrm{~h}$ post-inoculation. The tissue samples were homogenized in RLT buffer ( $\mathrm{RNe}$ asy ${ }^{\circledR}$ Mini Kit, QIAGEN Corporation, Germany) and disrupted using a Tissue Lyser II (QIAGEN Corporation, Germany).

Viral loads in clarified supernatants and blood were determined using a One-Step PrimeScript RT-PCR Kit (TAKARA, Japan). Viral RNA was extracted using an RNeasy ${ }^{\circledR}$ Mini Kit (QIAGEN Corporation, Germany) according to the manufacturer's instructions. A pair of primers (CVA16YGF, GGGAATTTCTTTAGCCGT GC; CVA16YGR, CCCATCAARTCAATGTCCC) and a hybridization probe (CVA16YGPB, FAM-ACA ATG CCC ACC ACG GGT ACA CA-BHQ1) were designed for the specific detection of CV-A16. The optimized thermal cycling protocol was as follows: reverse transcription program $\left(42^{\circ} \mathrm{C}\right.$ for $\left.30 \mathrm{~min}\right)$, denaturation program $\left(95^{\circ} \mathrm{C}\right.$ for $2 \mathrm{~min}$ ), amplification program repeated 40 times $\left(95^{\circ} \mathrm{C}\right.$ for $5 \mathrm{~s}$ followed by $55^{\circ} \mathrm{C}$ for $35 \mathrm{~s}$ ), and fluorescence measurement of each cycle at $55^{\circ} \mathrm{C}$ [25].

\section{Passive protection by neutralizing antibodies}

To confirm the protective efficacy of the anti-VLP serum (NT titre, 1:256) against CV-A16 challenge in vivo, four lethal viral challenge experiments were carried out.

Neonatal ICR mice (10-15 per group) less than $48 \mathrm{~h}$ old were inoculated i.p. with $50 \mu \mathrm{L}$ of serially tenfold diluted (1- to 1000 -fold dilutions) anti-VLP or $50 \mu \mathrm{L}$ of negative serum (heat-treated at $56{ }^{\circ} \mathrm{C}$ for $30 \mathrm{~min}$ prior to use), followed by the i.c. administration of $1000 \mathrm{LD}_{50}$ of CV-A16 521-01T at $1 \mathrm{~h}$ post-inoculation. Mortality and clinical symptoms were then monitored and recorded daily until 21 days post-challenge [24].

In another experiment, $1000 \mathrm{LD}_{50}$ of 521-01T was first neutralized by serial dilution with anti-VLP (1- to 1000fold dilutions) or negative serum (heat-treated at $56{ }^{\circ} \mathrm{C}$ for 30 min prior to use) in equal volumes in vitro. ICR mice less than $48 \mathrm{~h}$ of age (10-15 per group) were inoculated i.c. with the mixtures described above. All mice were monitored daily for clinical symptoms or death until 21 days post-inoculation.

Finally, the protective efficacy of maternal antibodies against homologous or heterologous CV-A16 challenge was studied. Female mice were allowed to mate after the second intranasal immunization as described above. The pups were born approximately 7 days after the third inoculation. After delivery, the pups were challenged i.c. with 521-01T (1000 LD $\mathrm{LD}_{50}$ /mouse) or NJ10-31 (1000 LD 50 mouse) on the first day after birth. The clinical symptoms and mortality of the challenged suckling mice were recorded daily. 


\section{Statistical analysis}

The data were analysed using GraphPad Prism software (version 5.00, GraphPad, La Jolla, CA, USA). Specific anti-CV-A16 IgG, neutralizing antibodies, IFN- $\gamma$ secreting splenic cells, and viral load values were evaluated by one-way ANOVA; Differences in the production of specific anti-CV-A16 IgA were evaluated by two-way ANOVA. Differences between two groups were evaluated by independent-samples $t$-tests, and survival rates were analysed by the Mantel-Cox log-rank test. The results are expressed as the mean \pm standard deviation (SD). A $P$-value of $<0.05$ was considered indicative of a statistically significant difference between values.

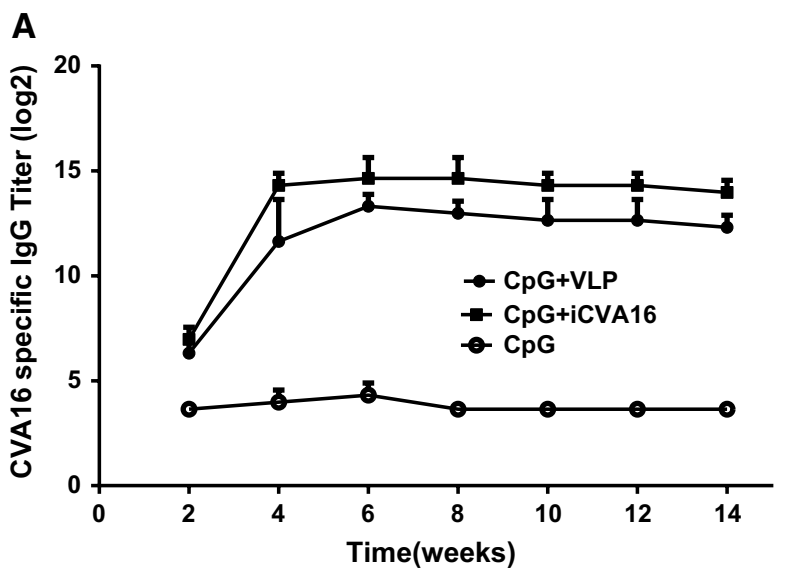

\section{Results}

\section{CV-A16-specific antibody responses elicited by VLPs in mice}

Inoculation with purified VLPs induced high levels of antiCV-A16-specific antibodies, the titres of which reached a maximum of $2^{12}$ approximately 2 weeks after the third immunization (Fig. 1A). The antibody titres were maintained at high levels for at least 14 weeks. In comparison, the inactivated CV-A16 induced slightly higher anti-CVA16 antibody titres in the fourth week; however, the timedependent changes in antibody titres and magnitudes after week 6 were similar (Fig. 1A). In addition, s-IgA was also detected in the lavage fluid from the respiratory tract, vagina and small intestine (Fig. 1B).

Neutralizing antibody titres in sera against VLPs and inactivated CV-A16 were determined. As shown in Fig. 1C,
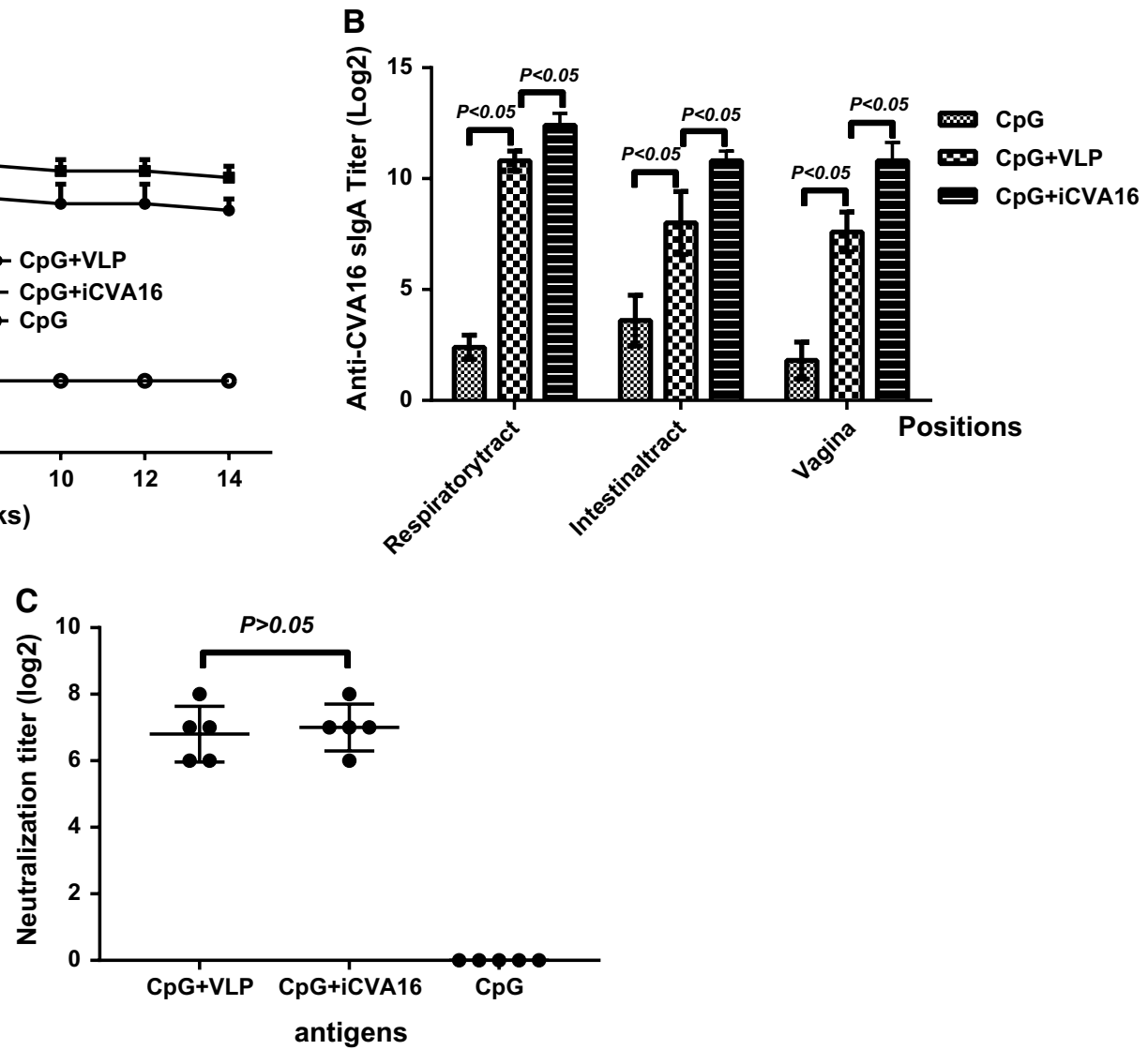

Fig. 1 Antibody responses following immunization of mice with CV-A16 VLPs and inactivated CV-A16. The geometric mean titres of (A) total IgG $(\log 2)$ (the difference in CV-A16-specific IgG titres between CV-A16 VLPs and inactivated CV-A16 was not significant at every time point), (B) s-IgA $(\log 2)$, and (C) neutralizing antibodies $(\log 2)$ against CV-A16 were determined by ELISA (for total specific
anti-CV-A16 IgG or s-IgA titration, the positive cutoff OD value was defined as 2.1 times that of normal mouse serum) and neutralization techniques. All values are presented as the mean \pm SD from three or five mice per group. A $P$-value $<0.05$ was considered statistically significant. iCVA16, inactivated coxsackievirus A16; VLP, virus-like particle; Ig, immunoglobulin 
the antibodies against both VLPs and inactivated CV-A16 neutralized the homologous CV-A16 521-01T strain efficiently with a mean titre of $1: 128$, whereas sera from the control group did not display any neutralization activity at all.

\section{Cellular immune responses elicited by VLP immunization}

ELISPOT analysis revealed a significantly larger number of CV-A16-specific IFN- $\gamma$-secreting cells in the spleens of mice vaccinated with inactivated CV-A16 or VLPs than in the spleens of $\mathrm{CpG}$ control mice (Fig. $2 ; P<0.05$ ). VLPimmunized mice had the same quantity of specific IFN- $\gamma$ producing $\mathrm{T}$ cells as the inactivated CV-A16-immunized mice. These results confirm that the cellular immune responses could be successfully induced by mucosal immunization with CV-A16 VLPs.

\section{The dose-related effects and $L D_{50}$ of the viruses}

ICR mice younger than $48 \mathrm{~h}$ were inoculated i.c. with serially diluted CV-A16 521-01T or NJ10-31. In the CV-A16 521-01T groups, the mice that were inoculated with a dose of $1.0 \times 10^{4} \mathrm{TCID}_{50}$ became sick (with tremors or hind limb paralysis) at 2-3 d.p.i., while the mice infected with a dose of $1.0 \times 10^{3} \mathrm{TCID}_{50}$ became sick at 3-4 d.p.i. These two infectious doses $\left(1.0 \times 10^{4}\right.$ and $\left.1.0 \times 10^{3} \mathrm{TCID}_{50}\right)$ caused death in all mice on different days postinfection ( 6 and 9 d.p.i.,

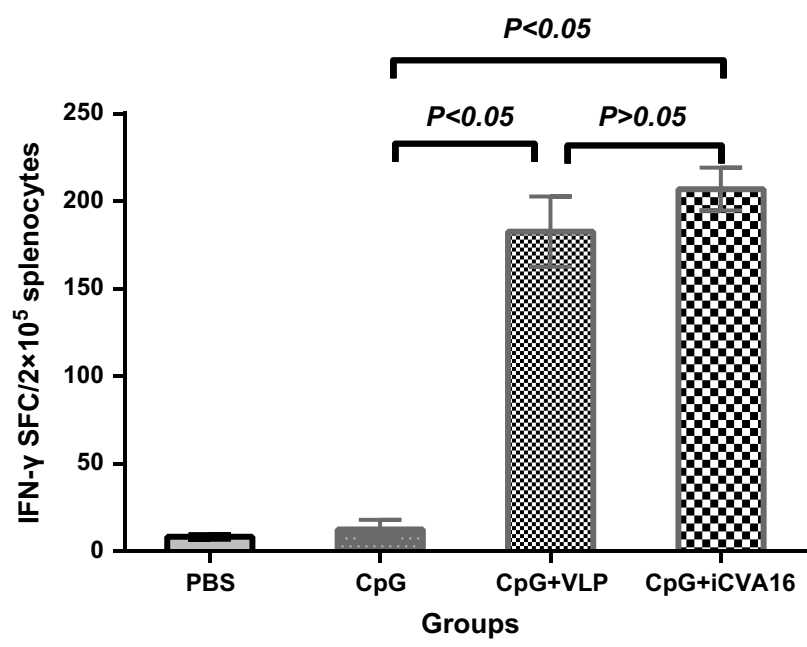

Fig. 2 Proliferation of splenocytes derived from mice immunized with CV-A16 VLPs or inactivated CV-A16. ELISPOT detection of IFN- $\gamma$-secreting $\mathrm{T}$ cells in response to CV-A16 in splenocytes from three mice from each group. Splenocytes were stimulated with purified VLPs or inactivated virions derived from CV-A16 521-01T. All data are expressed as the mean \pm SD. iCVA16, inactivated coxsackievirus A16; VLP, virus-like particle; SFC, spot-forming cells, IFN, interferon respectively). The mice infected with a dose of $1.0 \times 10^{2}$ $\mathrm{TCID}_{50}$ became sick at 6-7 d.p.i., and only one mouse survived until 15 d.p.i. In the mice infected with doses of $1.0 \times 10^{1}$ and $1.0 \mathrm{TCID}_{50}$, the mortality rates were $62.5 \%$ and $25 \%$, respectively; some mice progressively recovered after 12 days.

In the CV-A16 NJ10-31 groups, the mortality rates of the mice infected with $1.0 \times 10^{4}, 1.0 \times 10^{3}$, and $1.0 \times 10^{2} \mathrm{TCID}_{50}$ were $87.5 \%, 100 \%$, and $25 \%$, respectively. The mice infected with $1.0 \times 10^{1}$ and 1.0 all survived, with some mice recovering progressively.

These results reveal significant dose-dependent effects of CV-A16 on the mortality of neonatal mice (521-01T, $\mathrm{LD}_{50}=6.5 \mathrm{TCID}_{50} ; \mathrm{NJ} 10-31, \mathrm{LD}_{50}=250 \mathrm{TCID}_{50}$ ).

To guarantee the reproducibility of the mouse model results, a dose of $1000 \mathrm{LD}_{50}$ was given as a lethal challenge. Repeated experiments revealed that all neonatal mice became sick at approximately 3 d.p.i., and all mice administered this dose died at approximately 5-6 d.p.i. The times and rates of morbidity were stable.

\section{Dynamic distribution of virus in mouse tissues after challenge}

The tissue viral loads of inoculated mice at different time points may indicate the characteristics of virus infection and tropism (Fig. 3). The virus was first detected in the brain $\left(10^{2.59}\right.$ copies $\left./ \mathrm{mg}\right)$, blood $\left(10^{3.05}\right.$ copies $\left./ \mu \mathrm{L}\right)$ and cardiac tissues $\left(10^{2.07}\right.$ copies $\left./ \mathrm{mg}\right)$ at $1 \mathrm{~h}$ after challenge. At twenty-four hours after inoculation, the virus was detected in the blood, brain, heart and hind limb skeletal muscle tissues $\left(10^{3.00}\right.$ copies $/ \mu \mathrm{L}, 10^{3.87}$ copies $/ \mathrm{mg}, 10^{3.03}$ copies $/ \mathrm{mg}$ and $10^{4.51}$ copies/mg, respectively). Seventy-two hours later, the virus titre reached a peak in all tissues, including muscle $\left(10^{8.82}\right.$ copies/ $\mathrm{mg})$, blood $\left(10^{6.77}\right.$ copies $\left./ \mu \mathrm{L}\right)$, lung $\left(10^{5.13}\right.$ copies $\left./ \mathrm{mg}\right)$, intestine $\left(10^{4.30}\right.$ copies $\left./ \mathrm{mg}\right)$, liver $\left(10^{5.04}\right.$ copies $\left./ \mathrm{mg}\right)$, brain $\left(10^{4.42}\right.$ copies $/ \mathrm{mg})$, spleen $\left(10^{4.98}\right.$ copies $\left./ \mathrm{mg}\right)$, and heart $\left(10^{4.43} \mathrm{cop}\right.$ ies $/ \mathrm{mg}$ ), except the kidney $\left(10^{3.39}\right.$ copies/mg). These results suggested that the virus spread throughout the entire body through the bloodstream and proliferated rapidly during the first 24-72 $\mathrm{h}$. The amount of virus did not absolutely increase after $72 \mathrm{~h}$ in some tissues (heart, lung, brain and spleen) and even decreased. In the control group, no virus was detected in any of the tissues.

The viral load in the hind limb skeletal muscles steadily increased over time and was maintained at a high level until the mice died. At $120 \mathrm{~h}$, the viral load in the hind limb skeletal muscles was approximately 4-log values greater than that in the other tissues except for the kidney. The viral load in the kidney steadily increased over time and was slightly lower than that in the hind limb skeletal muscle. This result indicates that CV-A16 521-01T has a strong tropism for 


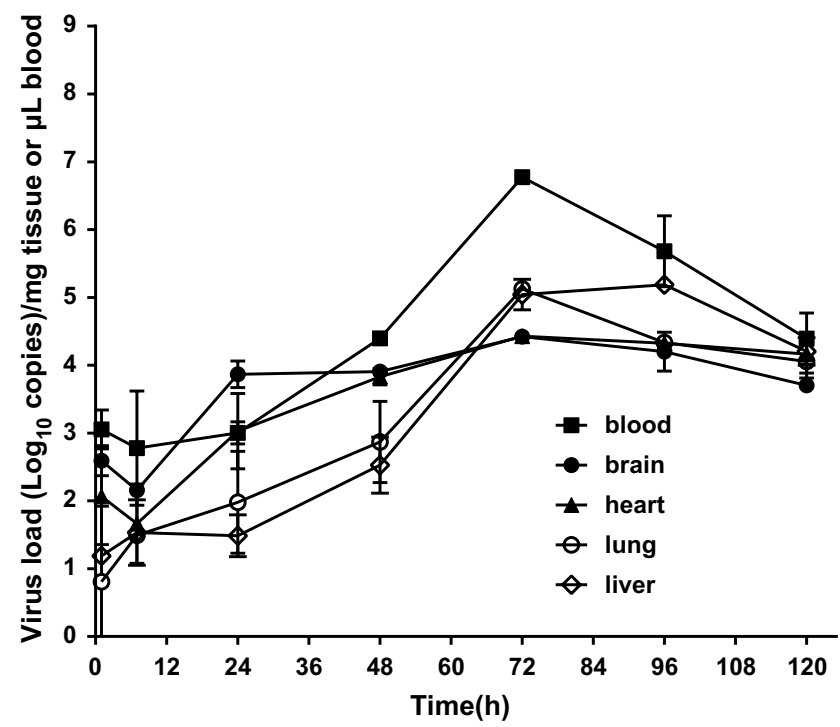

Fig. 3 Mean tissue viral loads in neonatal ICR mice infected with CV-A16 521-01T. Neonatal ICR mice less than two days old were inoculated i.c. with CV-A16 521-01T (1000 LD 50 /mouse). Viral loads in blood, brain, heart, lung, liver, spleen, intestine, kidney and skeletal muscle samples from the infected mice were determined using

skeletal muscle tissues, which may be the major location for viral replication.

\section{Sera from VLP-immunized mice protect neonatal mice against lethal challenge}

The first experiment showed that passive immunization with anti-VLP serum protected neonatal ICR mice against CV-A16 challenge. After 3 days, the mice that received the negative control or 1000-fold or 100 -fold diluted antisera started to show disease symptoms, such as inactivity, limb weakness, and even limb paralysis, and then began to die (Fig. 4A). All mice in the 1000-fold-diluted-antiserum groups were dead at 5 d.p.i., while all mice in the 100-fold diluted-group were dead at 7 d.p.i. Mice in the 10-folddiluted group began to show disease symptoms at 5 d.p.i. and began to die at 6 d.p.i. Some mice in this group suffered from mild symptoms and gradually recovered, with a survival rate of $50 \%$ at the end of the 21-day period (Fig. 4A). In contrast, all of the mice treated with undiluted anti-VLP sera remained healthy and survived throughout the course of the experiment. According to the survival rates of the different groups, a significant antibody-concentration-dependent effect on the protection rate was observed. The anti-VLP serum at tenfold dilution protected half of the neonatal ICR mice against lethal challenge.

In vitro neutralization results showed that the mice in the negative control and 1000-fold-diluted-antisera groups were all dead at 6 d.p.i.; they became sick and started to die at 3

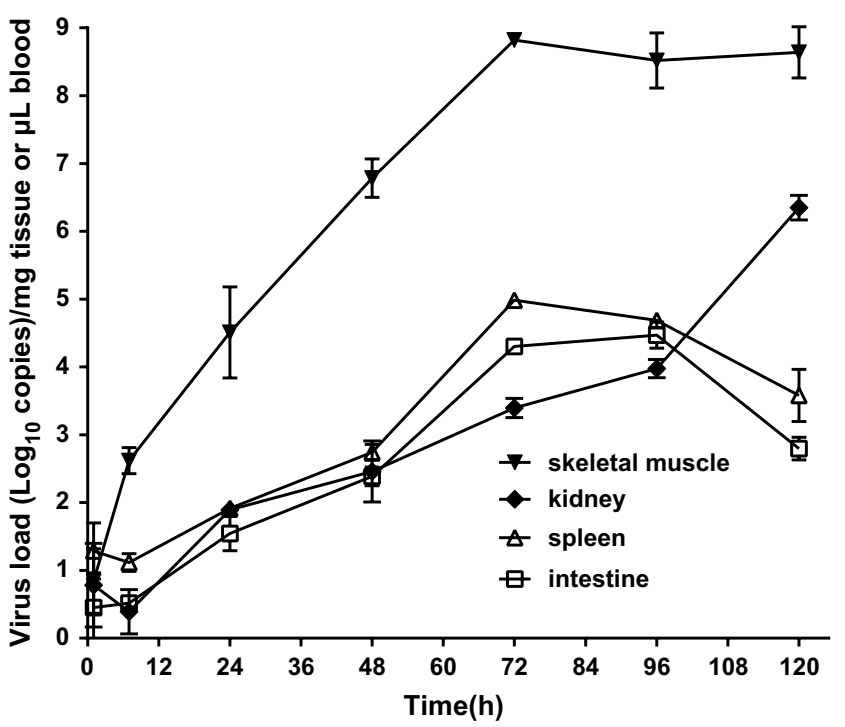

real-time quantitative reverse transcription PCR. Samples were collected at the indicated time points after the mice were sacrificed. The results are expressed as the mean viral $\operatorname{load}\left(\log _{10}\right.$ copies) per milligram of tissue or per microliter of blood \pm SD (three mice per time point) and are based on three independent experiments

d.p.i. (Fig. 4B). The mice in the 100-fold-diluted-antisera groups were all dead at 7 d.p.i.; they became sick at 3 d.p.i. and began to die at 4 d.p.i. The survival rate of the mice that received anti-VLP serum at a dilution of $1: 10$ was $50 \%$; these mice began to die at 8 d.p.i., and the rest recovered at 14 d.p.i. The mice receiving undiluted anti-VLP serum remained healthy throughout the course of the experiment.

Compared to control mice inoculated with culture medium, the mortality rate of mice receiving the anti-VLP serum was significantly reduced $(P<0.001)$. CV-A16 521$01 \mathrm{~T}$ pretreated with anti-VLP serum in vitro lost its lethal effect on neonatal mice.

The mortality of neonatal mice challenged with CV-A16 was prevented by maternal antibodies.

In this study, both CV-A16 VLPs and inactivated CV-A16 induced a good humoral immune response in adult mice, with neutralization titres ranging from 64 to 256 one week after the second boost. To assess the protective efficacy of maternal antibodies against challenge with homologous or heterologous CV-A16 strains, neonatal ICR mice from immunized mothers were challenged intracerebrally with CV-A16 521-01T or NJ 10-31.

As shown in Fig. $4 \mathrm{C}$ and $\mathrm{D}$, all the inoculated pups born to PBS-immunized mothers displayed a range of clinical symptoms (hunched back, wasting, and even hind limb paralysis) after 3 days of infection, followed by death beginning on day 4; all the mice were dead after 7 days. In contrast, all of the neonatal mice born to VLP-immunized mothers survived both the CV-A16 521-01T and 

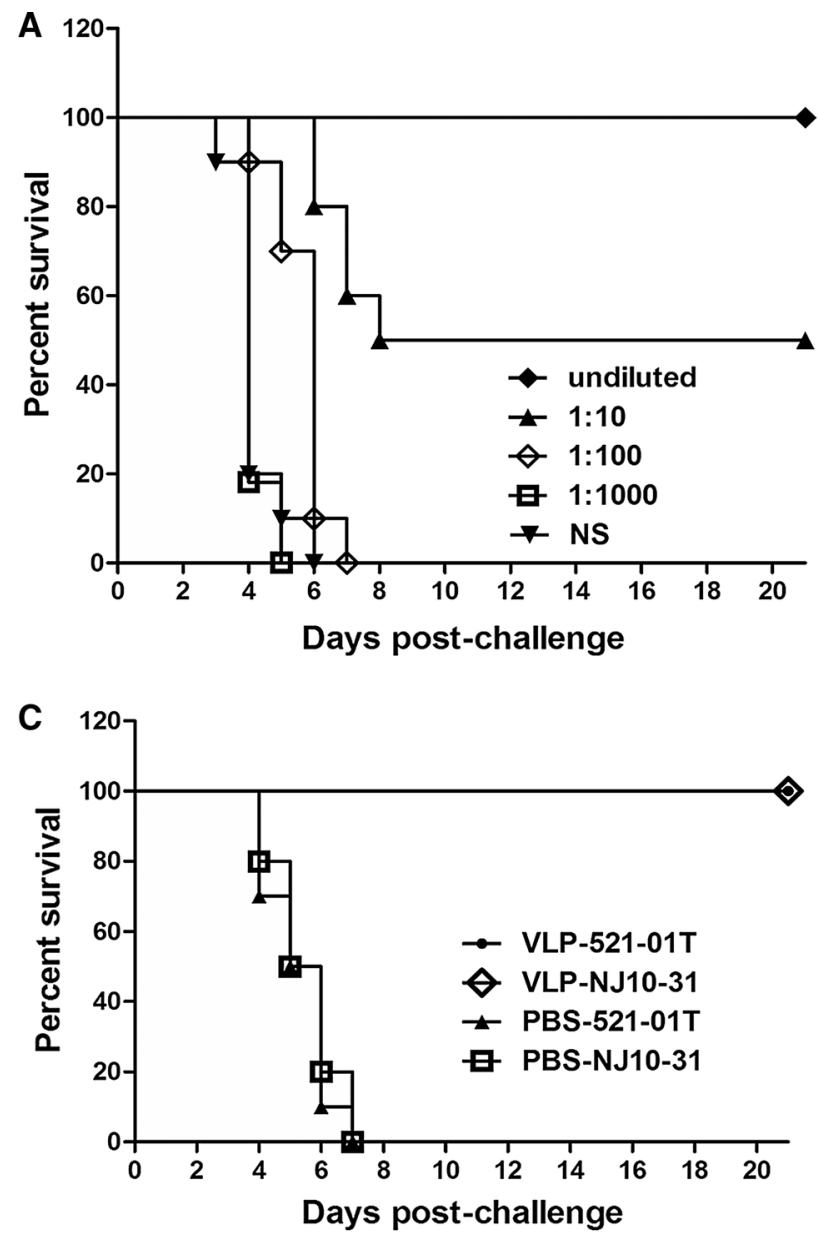

Fig. 4 Sera or maternally transferred antibodies from VLP-immunized mice conferred protective effects against lethal CV-A16 challenge in neonatal mice. (A) Passive immunization with serum induced by CV-A16 VLP immunization protected neonatal mice against CV-A16 challenge in vivo. Neonatal ICR mice (10-15 per group) less than $48 \mathrm{~h}$ old were inoculated i.p. with $50 \mu \mathrm{L}$ of serially tenfold diluted (1- to 1000-fold dilutions) anti-CV-A16 VLP serum (NT titre, 1: 256) or $50 \mu \mathrm{L}$ of negative serum (heat-treated at $56{ }^{\circ} \mathrm{C}$ for $30 \mathrm{~min}$ prior to use), followed by the i.c. administration of 1000 $\mathrm{LD}_{50}$ of CV-A16 521-01T at $1 \mathrm{~h}$ post-inoculation. The survival of the neonatal mice was then monitored and recorded daily until 21 days after challenge. The differences between each antiserum group and the control group were evaluated by the Mantel-Cox log-rank test. (B) The lethal effect of CV-A16 on neonatal mice could be significantly neutralized by pretreatment with anti-CV-A16 serum in vitro before challenge; $1000 \mathrm{LD}_{50}$ of 521-01T was first neutralized with an equal volume of 1- to 1000-fold serially diluted anti-VLP serum or negative serum (heat-treated at $56{ }^{\circ} \mathrm{C}$ for $30 \mathrm{~min}$ prior to use) in vitro. Neo-

NJ10-31 challenge and grew normally over the course of infection, except for one neonatal mouse in the NJ1031 group, which had mild limb paralysis. These results show that maternal antibodies completely protected the pups against challenge with a homologous or heterologous CV-A16 strain (Fig. 4C and D). These results also

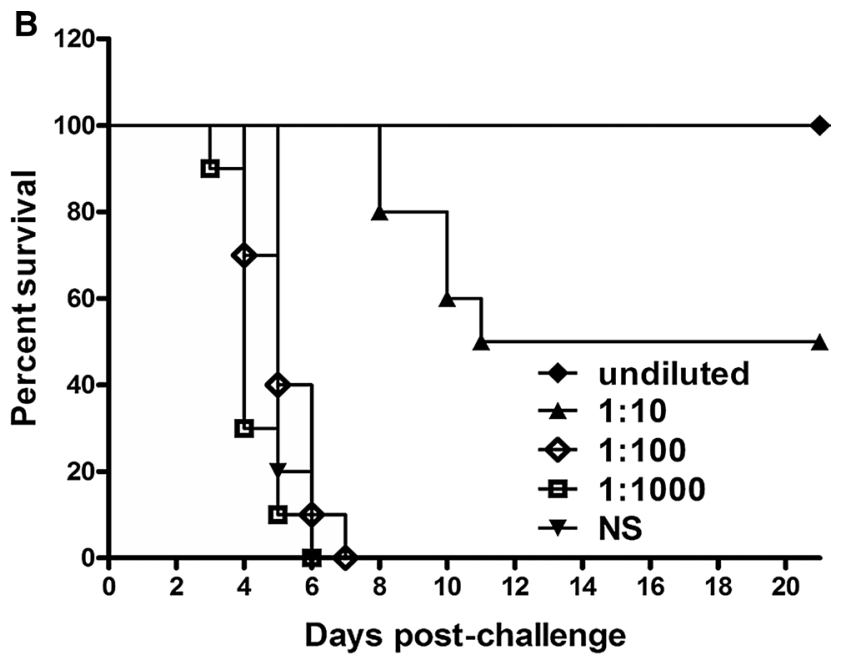

D

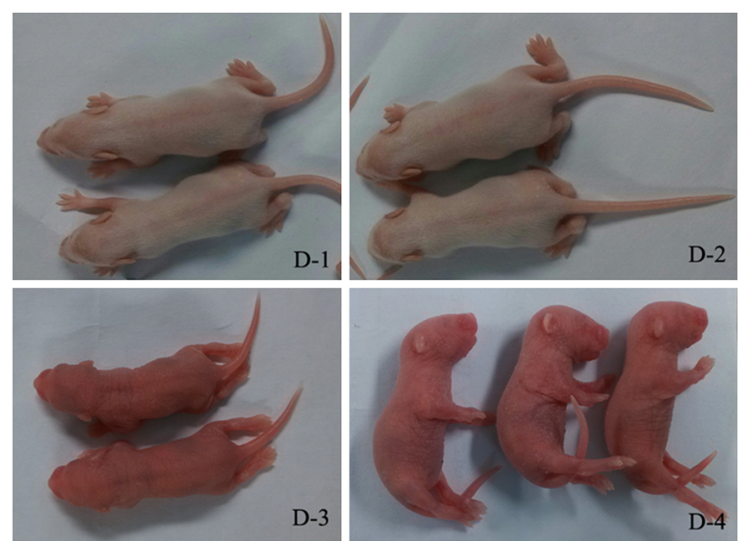

natal ICR mice less than $48 \mathrm{~h}$ old $(10 \sim 15$ per group) were treated i.c. with the mixtures described above. All mice were monitored daily until 21 days after the challenge. The Mantel-Cox log-rank test was used to compare the survival of neonatal mice between each antiserum group and the control group. (C) Maternal antibodies produced by CV-A16 VLP immunization protected newborn mice against lethal challenge. The female mice were allowed to mate after the second immunization as described in Materials and methods. The pups were born approximately 7 days after the third inoculation. After delivery, the pups were challenged i.c. with CV-A16 521-01T (1000 $\mathrm{LD}_{50}$ / mouse) and NJ10-31 (1000 LD 50 /mouse) on the first day after birth. The mortality of the challenged suckling mice was recorded daily after infection. The Mantel-Cox log-rank test was used to evaluate the survival of pups between each maternal CV-A16 VLP immunization group and the PBS control group at 21 d.p.i. (D) Representative images showing symptoms of the pups born to VLP-immunized female mice (D1 and D2) and PBS-immunized female mice (D3 and D4) after intracerebral challenge

indicate that the passive protection conferred by CV-A16 VLP immunization could be passed from the mothers to the neonates. These results reveal that the mortality rate of neonatal mice from mothers immunized with CV-A16 VLPs was significantly reduced (Mantel-Cox log-rank test, $P<0.0001)$. 


\section{Discussion}

Immunization with CV-A16 VLPs by injection has been shown to induce high neutralizing antibody titres and provide effective protection against $\mathrm{CV}-\mathrm{A} 16$ infection [18-20]. In this study, the immunogenicity of the CV-A16 VLP vaccine candidate when administered intranasally was evaluated. CV-A16 VLPs are considered excellent immunogens, as they not only induce high titres of neutralizing antibodies that protect against lethal viral challenge but also induce the production of s-IgA antibodies, demonstrating that immunization via the mucosal route generates both mucosal and systemic immune responses. The immune system component of mucosal surfaces is the first line of host defence against invading pathogens. The mucosal surface is the initial site of CV-A16 infection; therefore, $\mathrm{s}-\operatorname{Ig} \mathrm{A}$ antibodies are well adapted to providing specific immune protection against CV-A16 on mucosal surfaces.

Vaccination via the nasal mucosa has been widely studied in animal models and in clinical investigations [26-29]. The nose is easily accessible, and the nasal cavity contains numerous dendritic cells (DCs), T cells and B cells, which are covered by an epithelial layer containing distinctive cells called microfold (M) cells [30]. The generation of an immune response in the respiratory tract is mediated by nasal-associated lymphoid tissue (NALT) [31].

The generation of s-IgA antibodies is most effectively accomplished by stimulating the common mucosal immune system. In this study, we found that nasal immunization induced IgA not only in the respiratory tract but also at other mucosal sites, such as the intestinal tract and vagina. Mucosa is widely distributed in the human body, covering the surface of the respiratory, intestinal and urinary tracts. Different parts of the mucosa can be accessed by lymphocyte homing, which results in a systemic immune response [32, 33].

$\mathrm{CpG}$ can induce the innate immune response through recognition by Toll-like receptor 9 on plasmacytoid dendritic cells and B cells [34]. CpG has been used as an excellent systematic and mucosal adjuvant that increases the production of antibodies [35-37]. In this study, CpG was used as an adjuvant for nasally delivered CV-A16 VLPs to induce mucosal as well as systemic serological and cellular immune responses. Consistently, intranasal immunization with CV-A16 VLPs and CpG induced the production of high titres of specific neutralizing antibodies as well as the secretion of s-IgA by the respiratory tract, intestinal tract, and vaginal mucosa.

VLPs are generally safer than the inactivated CV-A16 virus due to their lacking viral nucleic acids and infectivity
[11]. Here, CV-A16 VLP immunization induced a level of CV-A16-specific neutralizing antibody production similar to that of immunization with inactivated CV-A16. These antibodies exhibited a strong neutralization capacity against the CV-A16 strains, which is consistent with previous reports $[18,38]$. Anti-VLP serum demonstrated excellent protective effects in challenge experiments with neonatal mice. Anti-VLP serum significantly reduced the mortality rate of the recipient neonatal mice. CV-A16 virus pretreated with anti-VLP serum in vitro lost its lethal activity in neonatal mice. The protective effect of maternally transferred anti-VLP antibodies was also evaluated in newborn mice. The maternally transferred antibodies provided $100 \%$ protection against challenge with 1000 $\mathrm{LD}_{50}$ of homologous or heterologous strains of CV-A16.

Neutralizing antibodies play a critical role in protection against CV-A16 infection; however, cellular immune responses are also important in human immunity $[17,39]$. In the present study, intranasal immunization with CV-A16 VLPs induced cellular immune responses similar to those achieved via intranasal immunization with inactivated CV-A16. These results demonstrate that immunization with CV-A16 VLPs by the mucosal route induces both cellular and humoral responses in mice.

In conclusion, the results presented here confirm the complete protective effect of neutralizing antibodies produced by immunization with the CV-A16 VLP vaccine through the mucosal route. These results represent valuable supplemental information for understanding the specific protective effect of CV-A16 VLPs and have important significance for the development of a safe and effective vaccine.

Author contributions $\mathrm{XC}$ and $\mathrm{WX}$ conceived and designed the study; $\mathrm{XC}$ performed the experiments; YZ performed the data analyses; NM performed the ELISPOT analyses; SZ and TJ performed the ELISAs and analysed and interpreted the data. The manuscript was written by XC and proofread by WX. All authors revised the manuscript and approved the final version.

Funding This work was supported by the Beijing Natural Science Foundation (Grant no. 7184208), the National Science and Technology Major Project of the Ministry of Science and Technology of China (Grant nos. 2018ZX10201002-003-009, 2017ZX10104001-005-010, 2018ZX10713002, and 2018ZX10713001-003), the Basic and Clinical Research Cooperation Project of Capital Medical University (Grant no. 17JL11), and the Research Training Fund of Capital Medical University (Grant no. PYZ2017012). The sponsors played no role in the study design, data analysis, manuscript preparation, or decision to publish.

\section{Compliance with ethical standards}

Conflict of interest The authors have no conflicts of interest to declare.

Animal and human rights statement The program for immunization with CV-A16 VLPs or inactivated CV-A16 combined with CpG ODN was approved by the Ethical Committee of the National Institute of 
Viral Disease Control and Prevention, China CDC. The animal care and use protocol in this study abided by "The Guidance on Treating Experimental Animals", which was promulgated by the Ministry of Science and Technology of China. No nonhuman primates were used in this study.

\section{References}

1. Liu W, Wu S, Xiong Y, Li T, Wen Z, Yan M, Qin K, Liu Y, Wu J (2014) Co-circulation and genomic recombination of coxsackievirus A16 and enterovirus 71 during a large outbreak of hand, foot, and mouth disease in Central China. PLoS One 9:e96051. https:// doi.org/10.1371/journal.pone.0096051

2. Liu Y, Wang X, Pang C, Yuan Z, Li H, Xue F (2015) Spatiotemporal analysis of the relationship between climate and hand, foot, and mouth disease in Shandong province, China, 20082012. BMC Infect Dis 15:146. https://doi.org/10.1186/s1287 9-015-0901-4

3. Ang LW, Koh BK, Chan KP, Chua LT, James L, Goh KT (2009) Epidemiology and control of hand, foot and mouth disease in Singapore, 2001-2007. Ann Acad Med Singap 38:106-112

4. Chen JF, Zhang RS, Ou XH, Chen FM, Sun BC (2014) The role of enterovirus 71 and coxsackievirus A strains in a large outbreak of hand, foot, and mouth disease in 2012 in Changsha, China. Int J Infect Dis 28:17-25. https://doi.org/10.1016/j.ijid.2014.07.024

5. Xing W, Liao Q, Viboud C, Zhang J, Sun J, Wu JT, Chang Z, Liu F, Fang VJ, Zheng Y, Cowling BJ, Varma JK, Farrar JJ, Leung GM, Yu H (2014) Hand, foot, and mouth disease in China, 200812: an epidemiological study. Lancet Infect Dis 14:308-318. https ://doi.org/10.1016/S1473-3099(13)70342-6

6. Du J, Wang X, Hu Y, Li Z, Li Y, Sun S, Yang F, Jin Q (2014) Changing aetiology of hand, foot and mouth disease in Linyi, China, 2009-2011. Clin Microbiol Infect 20:O47-O49. https:// doi.org/10.1111/1469-0691.12301

7. Sun LM, Wu SL, Tan XH, Li H, Yang F, Zeng HR, Zheng HY, Liu L, He JF (2018) Epidemiological characteristics of Coxsackie virus A16 caused hand foot and mouth disease cases in Guangdong province, 2012-2016. Zhonghua Liu Xing Bing Xue Za Zhi 39:342-346. https://doi.org/10.3760/cma.j.i ssn.0254-6450.2018.03.018

8. Yee PTI, Laa Poh C (2017) Impact of genetic changes, pathogenicity and antigenicity on Enterovirus-A71 vaccine development. Virology 506:121-129. https://doi.org/10.1016/j.virol .2017.03.017

9. Mao Q, Wang Y, Yao X, Bian L, Wu X, Xu M, Liang Z (2014) Coxsackievirus A16: epidemiology, diagnosis, and vaccine. Hum Vaccin Immunother 10:360-367. https://doi.org/10.4161/ hv. 27087

10. Wang CY, Li LuF, Wu MH, Lee CY, Huang LM (2004) Fatal coxsackievirus A16 infection. Pediatr Infect Dis J 23:275-276

11. Chackerian B (2007) Virus-like particles: flexible platforms for vaccine development. Expert Rev Vaccines 6:381-390. https:// doi.org/10.1586/14760584.6.3.381

12. Li HZ, Gang HY, Sun QM, Liu X, Ma YB, Sun MS, Dai CB (2004) Production in Pichia pastoris and characterization of genetic engineered chimeric HBV/HEV virus-like particles. Chin Med Sci J 19:78-83

13. McCormack PL (2014) Quadrivalent human papillomavirus (types $6,11,16,18)$ recombinant vaccine (gardasil $((\mathrm{R})))$ : a review of its use in the prevention of premalignant anogenital lesions, cervical and anal cancers, and genital warts. Drugs 74:1253-1283. https ://doi.org/10.1007/s40265-014-0255-z
14. Siddiqui MA, Perry CM (2006) Human papillomavirus quadrivalent (types $6,11,16,18$ ) recombinant vaccine (Gardasil). Drugs 66:1263-1271 (discussion 1272-1263)

15. Szarewski A (2010) HPV vaccine: cervarix. Expert Opin Biol Ther 10:477-487. https://doi.org/10.1517/14712591003601944

16. Chung YC, Ho MS, Wu JC, Chen WJ, Huang JH, Chou ST, Hu YC (2008) Immunization with virus-like particles of enterovirus 71 elicits potent immune responses and protects mice against lethal challenge. Vaccine 26:1855-1862

17. Cao L, Mao F, Pang Z, Yi Y, Qiu F, Tian R, Meng Q, Jia Z, Bi S (2015) Protective effect of enterovirus71 (EV71) viruslike particle vaccine against lethal EV71 infection in a neonatal mouse model. Mol Med Rep 12:2473-2480. https://doi.org/10.3892/ mmr.2015.3680

18. Zhang C, Liu Q, Ku Z, Hu Y, Ye X, Zhang Y, Huang Z (2016) Coxsackievirus A16-like particles produced in Pichia pastoris elicit high-titer neutralizing antibodies and confer protection against lethal viral challenge in mice. Antivir Res 129:47-51. https://doi.org/10.1016/j.antiviral.2016.02.011

19. Feng Q, He Y, Lu J (2016) Virus-like particles produced in Pichia pastoris induce protective immune responses against coxsackievirus A16 in mice. Med Sci Monit Int Med J Exp Clin Res 22:3370-3382

20. Chen XP, Mao NY, Zhang Y, Xie ZD, Xu WB (2014) Preparation and immunogenicity of virus-like particles of Coxsackievirus A16. Chin J Biol 27:1361-1374

21. Chen XP, Tan XJ, Zhang Y, Xu WB (2014) Immunoprotective effect of inactivated coxsackievirus A16 vaccine in mice. Bing Du Xue Bao 30:226-232

22. Melnick JL, Wimberly IL (1985) Lyophilized combination pools of enterovirus equine antisera: new LBM pools prepared from reserves of antisera stored frozen for two decades. Bull World Health Organ 63:543-550

23. Reed LJ, Muench H (1938) A simple method of estimating 50\% end-points. Am J Hyg 27:493-497

24. Mao Q, Wang Y, Gao R, Shao J, Yao X, Lang S, Wang C, Mao P, Liang Z, Wang J (2012) A neonatal mouse model of coxsackievirus A16 for vaccine evaluation. J Virol 86:11967-11976. https://doi.org/10.1128/JVI.00902-12

25. Cui A, Xu C, Tan X, Zhang Y, Zhu Z, Mao N, Lu Y, Xu W (2013) The development and application of the two real-time RT-PCR assays to detect the pathogen of HFMD. PLoS One 8:e61451. https://doi.org/10.1371/journal.pone.0061451

26. Illum L, Jabbal-Gill I, Hinchcliffe M, Fisher AN, Davis SS (2001) Chitosan as a novel nasal delivery system for vaccines. Adv Drug Deliv Rev 51:81-96

27. Kemble G, Greenberg H (2003) Novel generations of influenza vaccines. Vaccine 21:1789-1795

28. Nantachit N, Sunintaboon P, Ubol S (2016) Responses of primary human nasal epithelial cells to EDIII-DENV stimulation: the first step to intranasal dengue vaccination. Virol J 13:142. https://doi.org/10.1186/s12985-016-0598-Z

29. Rose MA, Zielen S, Baumann U (2012) Mucosal immunity and nasal influenza vaccination. Expert Rev Vaccines 11:595-607. https://doi.org/10.1586/erv.12.31

30. Wu HY, Nguyen HH, Russell MW (1997) Nasal lymphoid tissue (NALT) as a mucosal immune inductive site. Scand J Immunol 46:506-513

31. Kuper CF, Koornstra PJ, Hameleers DM, Biewenga J, Spit BJ, Duijvestijn AM, van Breda Vriesman PJ, Sminia T (1992) The role of nasopharyngeal lymphoid tissue. Immunol Today 13:219-224

32. Brandtzaeg P, Johansen FE (2005) Mucosal B cells: phenotypic characteristics, transcriptional regulation, and homing properties. Immunol Rev 206:32-63. https://doi.org/10.111 1/j.0105-2896.2005.00283.x 
33. Kiyono H, Fukuyama S (2004) NALT-versus Peyer's-patch-mediated mucosal immunity. Nat Rev Immunol 4:699-710. https://doi. org/10.1038/nri1439

34. Birk R, Aderhold C, Hormann K, Wenzel A, Kramer B, Eschenhagen T, Sommer JU (2016) CpG-oligodeoxynucleotides in chronic rhinosinusitis cell culture. In Vivo 30:47-52

35. Meng Z, Zhang X, Pei R, Zhang E, Kemper T, Vollmer J, Davis HL, Glebe D, Gerlich W, Roggendorf M, Lu M (2016) Combination therapy including $\mathrm{CpG}$ oligodeoxynucleotides and entecavir induces early viral response and enhanced inhibition of viral replication in a woodchuck model of chronic hepadnaviral infection. Antivir Res 125:14-24. https://doi.org/10.1016/j.antiv iral.2015.11.001

36. Xiang XX, Zhou XQ, Wang JX, Xie Q, Cai X, Yu H, Zhou HJ (2011) Effects of CpG-ODNs on phenotype and function of monocyte-derived dendritic cells in chronic hepatitis B. World J Gastroenterol 17:4825-4830. https://doi.org/10.3748/wjg.v17. i43.4825

37. Lin YL, Chow YH, Huang LM, Hsieh SM, Cheng PY, Hu KC, Chiang BL (2018) A CpG-adjuvanted intranasal enterovirus 71 vaccine elicits mucosal and systemic immune responses and protects human SCARB2-transgenic mice against lethal challenge. Sci Rep 8:10713. https://doi.org/10.1038/s41598-018-28281-5

38. Liu Q, Yan K, Feng Y, Huang X, Ku Z, Cai Y, Liu F, Shi J, Huang $\mathrm{Z}$ (2012) A virus-like particle vaccine for coxsackievirus A16 potently elicits neutralizing antibodies that protect mice against lethal challenge. Vaccine 30:6642-6648. https://doi.org/10.1016/j. vaccine.2012.08.071

39. Emeny RT, Wheeler CM, Jansen KU, Hunt WC, Fu TM, Smith JF, MacMullen S, Esser MT, Paliard X (2002) Priming of human papillomavirus type 11-specific humoral and cellular immune responses in college-aged women with a virus-like particle vaccine. J Virol 76:7832-7842. https://doi.org/10.1128/ jvi.76.15.7832-7842.2002

Publisher's Note Springer Nature remains neutral with regard to jurisdictional claims in published maps and institutional affiliations. 